\title{
Photonic subsystems for optical packet/burst switches based on heterogeneous SOI and III-V integration
}

\author{
Dries Van Thourhout ${ }^{1}$, Martijn Tassaert ${ }^{1}$, Peter De Heyn ${ }^{1}$, Oded Raz $^{2}$, Nicola Calabretta ${ }^{2}$, H.J.S. Dorren ${ }^{2}$, \\ Günther Roelkens ${ }^{1}$ \\ ${ }^{\text {I}}$ Photonics Research Group, Department of Information Technology, Ghent University - imec, B-9000 Ghent, Belgium \\ ${ }^{2}$ Department of Electrical Engineering, Technical University of Eindhoven (TU/e), 5600MB Eindhoven, The Netherlands \\ email:dries.vanthourhout@ugent.be
}

\begin{abstract}
In this paper we describe how high-quality silicon photonic ICs and III-V membrane switches integrated on this platform can be used to build photonic subsystems for optical packet switches.

OCIS codes: (130.0130) Optical integration; (200.0200) Optics in Computing
\end{abstract}

\section{Introduction}

The quality of passive optical devices fabricated in high index contrast silicon waveguide platforms has improved steadily over the last years. Low loss ( $<1 \mathrm{~dB} / \mathrm{cm}$ for rib waveguides, $<0.15 \mathrm{~dB} / \mathrm{cm}$ for ridge waveguides [1]), highly wavelength selective filters $(\mathrm{Q}>0.5$ Million [2]) and good AWG demultiplexers (crosstalk $>25 \mathrm{~dB}[3])$ are all available now. In addition using our well established III-V on silicon heterogeneous integration platform we demonstrated optical switches with high extinction ratio. Together these functionalities allow realizing several of the building blocks required for complex optical packet switches on a very compact footprint.

\section{Optical label extractor}

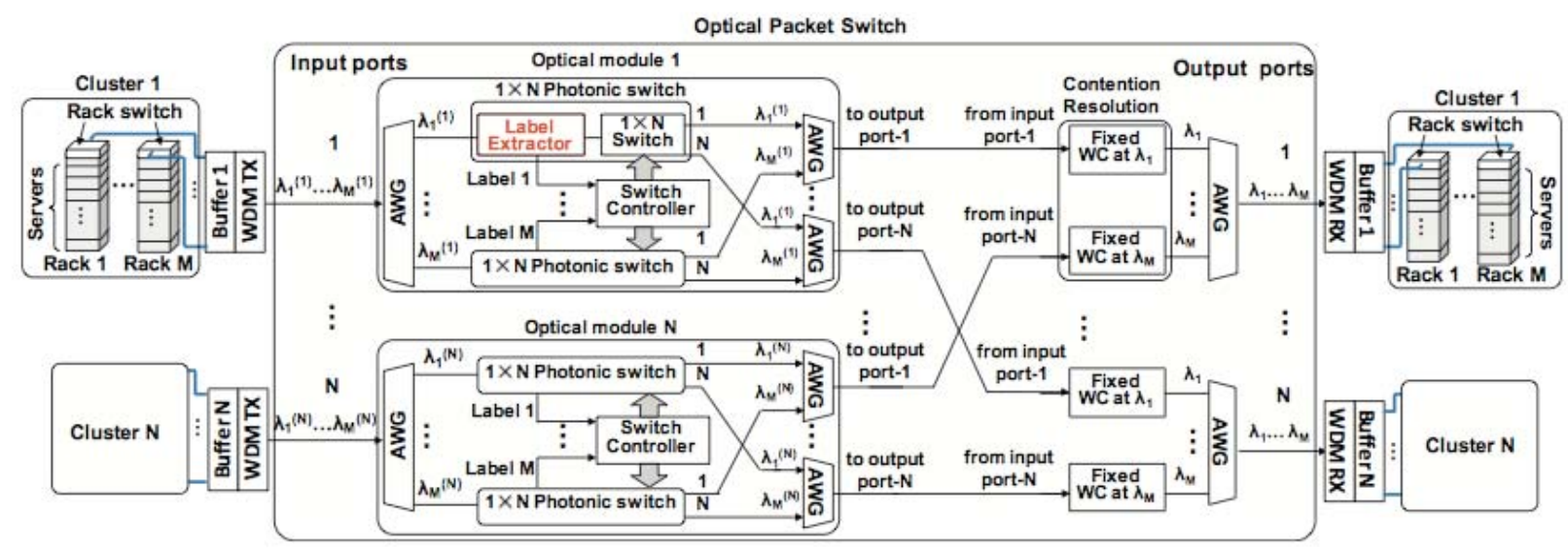

Fig. 1 Example of a modular WDM optical packet switch architecture (from [4])

Fig. 1 shows the schematic of a proposed optical packet switch [4]. An important building block within this switch is the optical label extractor, which separates the narrowband labels determining the packet address from the payload. We successfully demonstrated such a label extractor using a chip consisting of 4 cascaded high-Q ring resonators [5]. Fig. 2a shows the spectrum of the packet arriving at the label extractor, including a 160GBit/s modulated packet and 4 narrowband labels encoded within the band. Fig. $2 b$ shows the superimposed spectra of the 4 labels after passing through the label extractor shown in Fig. 2c. Extensive bit error measurements were carried out on the labels as well as on the transmitted payload. 

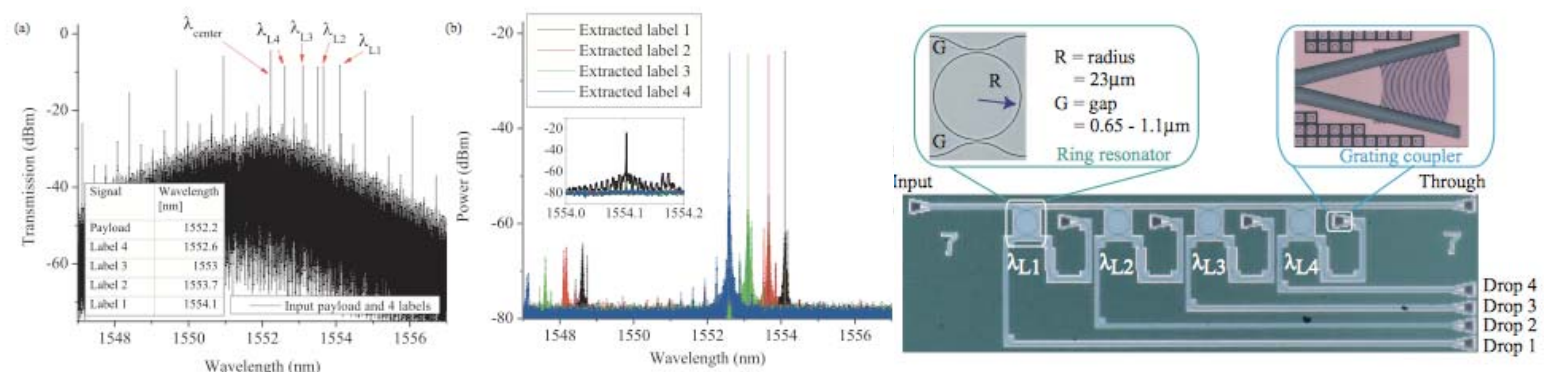

Fig. 2 (a) Optical spectra at the input spectrum of the label extractor consisting of a $160 \mathrm{~Gb} / \mathrm{s}$ modulated payload signal centered at $1552.2 \mathrm{~nm}$ and four $1.3 \mathrm{~Gb} / \mathrm{s}$ modulated label signals. (b) the output spectra of the four labels measured at the drop ports of the label extractor shown in (c). (taken from [5])

\section{MIPS: Membrane InP swich}

Using our III-V on silicon heterogeneous integration platform we developed switches with high performance. As shown in Fig. 3a,b these switches consist of a thin InP membrane containing 3 InGaAsP quantum wells bonded on an SOI platform [10][9][8]. In the off condition this device is highly absorbing in the wavelength range 1530nm1570nm. However, when pumped with an optical beam the switches become transparent Fig. 3c. The high optical confinement in the InP membrane thereby strongly reduces the required pump power.
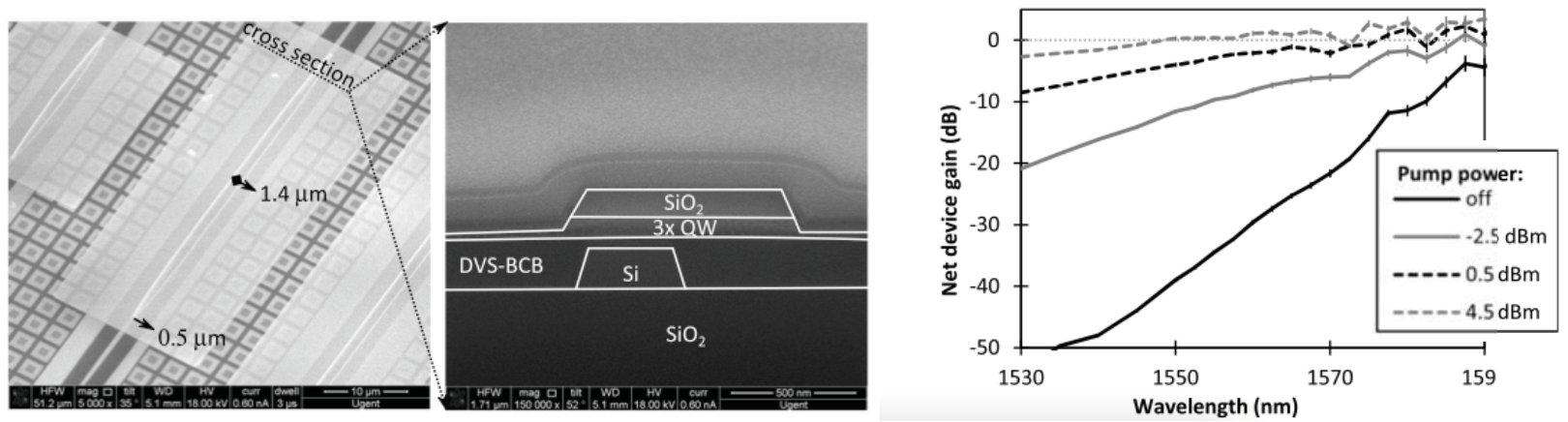

Fig. 3 a) Top view of InP Membrane Switch. b) Cross-section. c) Transmission when optically pumped (from [8])

Next we integrated these Membrane InP Switches (MIPS) into a 1 x 4 network containing splitters and a wavelength selective AWG as shown in Fig. 4a. Through choice of the wavelength of the pump beam 1 of the 4 MIPS is selected and pumped to transparency, while the other paths remain blocked [6][7]. Fig. 4b shows the transmission of two packets through the system (with the packet in between the two packets transmitted being blocked).
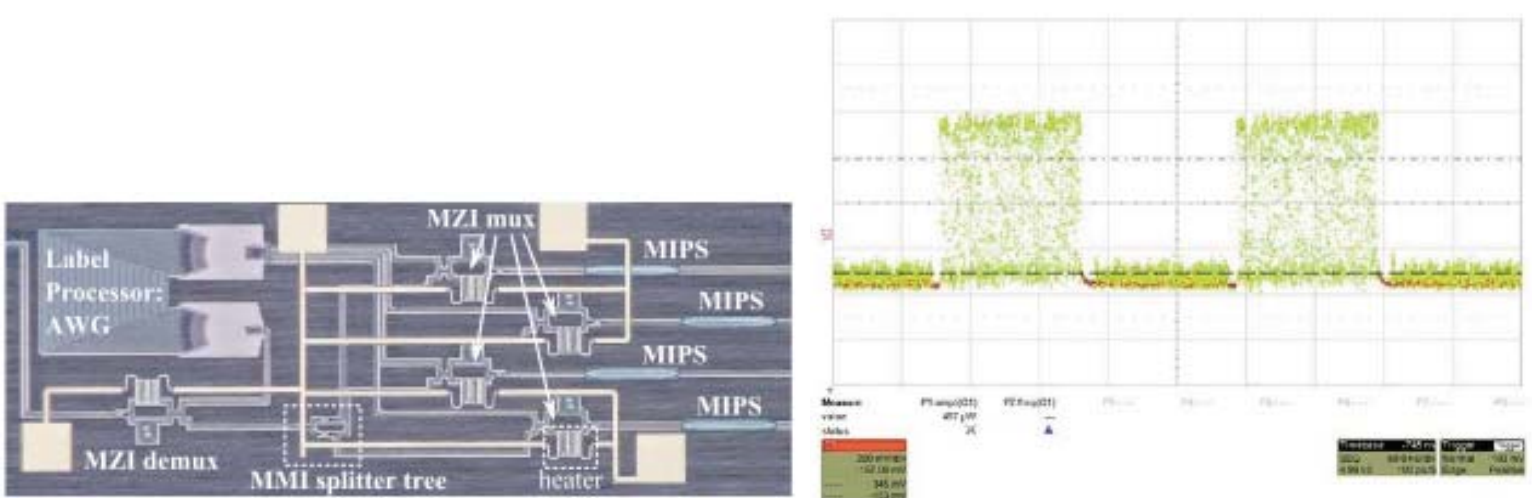

Fig. 4 a) Top view of $1 \times 4$ MIPS powered switch. b) Detected data signal at the AC receiver. One out of two packets is being blocked by the MIPS. (from [6]) 


\section{Acknowledgements}

The authors acknowledge support from the ERC-project ULPPIC, the EU-project FP7 LIGHTNESS and of the IAPPhoton network.

\section{References}

[1] S. Selvaraja, P. De Heyn, G. Winroth, P. Ong, G. Lepage, C. Cailler, A. Rigny, K. Bourdelle, W. Bogaerts, D. Van Thourhout, J. Van Campenhout, P. Absil, "Highly uniform and low-loss passive silicon photonics devices using a 300mm CMOS platform", Optical Fiber Communication Conference (OF2014), United States, p.Th2A.33 (2014)

[2] P. De Heyn, D. Vermeulen, T. Van Vaerenbergh, B. Kuyken, D. Van Thourhout, "Ultra-high Q and finesse all-pass microring resonators on Silicon-on-Insulator using rib waveguides", 16th European Conference on Integrated Optics and technical exhibition, Spain, p.paper 195 (2012) P. De Heyn, J. Luo, A. Trita, S. Pathak, S. Di Lucente, H. Dorren, N. Calabretta, D. Van Thourhout, “A Compact Integrated 40Gb/s Packet Demultiplexer and Label Extractor on Silicon-on-Insulator for an Optical Packet Switch", 39th European Conference and Exhibition on Optical Communication (ECOC), United Kingdom, p.paper Th.2.A.5 (2013)

[3] S. Pathak, M. Vanslembrouck, P. Dumon, D. Van Thourhout, P. Verheyen, G. Lepage, P. Absil, W. Bogaerts, "Effect of mask discretization on performance of silicon arrayed waveguide gratings", Photonics Technology Letters, 26(7), p.718-721 (2014)

[4] S. Di Lucente, J. Luo, R. P. Centelles, A. Rohit, S. Zou, K. a. Williams, H. J. S. Dorren, and N. Calabretta, "Numerical and experimental study of a high port-density WDM optical packet switch architecture for data centers." Optics express, vol. 21, no. 1, pp. $263-9$, Jan. 2013.

[5] P. De Heyn, J. Luo, S. Di Lucente, N. Calabretta, H.J.S Dorren, D. Van Thourhout, "In-Band Label Extractor Based on Cascaded Si Ring Resonators Enabling 160Gb/s Optical Packet Switching Modules", Journal of Lightwave Technology, (2014)

[6] M. Tassaert, G. Roelkens, H.J.S. Dorren, O. Raz, "Optically Reconfigurable 1x4 Remote Node Switch for Access Networks", Journal of Lightwave Technology, (2013)

[7] M. Tassaert, H.J.S Dorren, G. Roelkens, O. Raz, "Passive InP regenerator integrated on SOI for the support of broadband silicon modulators", Optics Express, 20(10), p.11383-11388 (2012)

[8] M. Tassaert, S. Keyvaninia, D. Van Thourhout, W.M.J. Green, Y. Vlasov, G. Roelkens, "An optically pumped nanophotonic InP/InGaAlAs optical amplifier integrated on a SOI waveguide circuit”, Optical and Quantum Electronics, 44(12), p.513-519 (2012)

[9] M. Tassaert, G. Roelkens, H.J.S. Dorren, D. Van Thourhout, O. Raz, "Bias-free, low power and optically driven membrane InP switch on SOI for remotely configurable photonic packet switches", Optics Express, 19(26), p.B817-B824 (2011)

[10] M. Tassaert, D. Van Thourhout, G. Roelkens, "Strategies to increase the modal gain in heterogeneously integrated III-V amplifiers on Silicon-On-Insulator", Optical and Quantum Electronics, p.683-689 (2011) 